\title{
Association Between the Level of Low-Density Lipoprotein Cholesterol and Coronary Atherosclerosis in Patients Who Have Undergone Coronary Computed Tomography Angiography
}

\author{
Hiroko Inoue $^{\mathrm{a}, \mathrm{c}}$, Yuhei Shiga ${ }^{\mathrm{b}, \mathrm{c}}$, Kohei Tashiro ${ }^{\mathrm{b}}$, Yuto Kawahira ${ }^{\mathrm{b}}$, Yasunori Suematsu ${ }^{\mathrm{b}}$, \\ Yoshiaki Idemoto $^{\mathrm{b}}$, Kanako Tano ${ }^{\mathrm{b}}$, Takashi Kuwano ${ }^{\mathrm{b}}$, Makoto Sugihara ${ }^{\mathrm{b}}$, \\ Hiroaki Nishikawa ${ }^{a}$, Yousuke Katsuda ${ }^{a}$, Shin-ichiro Miura ${ }^{a}$, b, d
}

\begin{abstract}
Background: Although the Japan Atherosclerosis Society Guidelines 2017 recommend lower levels of low-density lipoprotein cholesterol (LDL-C, $<70 \mathrm{mg} / \mathrm{dL}$ or $\leq 100 \mathrm{mg} / \mathrm{dL}$ ) to prevent secondary cardiovascular events, we cannot conclude that a low level of LDL-C prevents primary cardiovascular events in patients with suspected coronary artery disease (CAD).

Methods: We registered 1,016 patients who were clinically suspected to have $\mathrm{CAD}$ and who underwent coronary computed tomography angiography (CCTA) for screening of coronary atherosclerosis. We excluded 350 patients who were receiving anti-lipidemic therapies and finally analyzed 666 patients. The patients were divided into three groups according to the LDL-C level: $<70 \mathrm{mg} / \mathrm{dL}$ ( $\mathrm{n}=25$, Low LDL-C), 70 - $99 \mathrm{mg} / \mathrm{dL}(\mathrm{n}=141$, Middle LDL-C), and $\geq 100 \mathrm{mg} /$ $\mathrm{dL}(\mathrm{n}=500$, High LDL-C). A $\geq 50 \%$ coronary stenosis was initially diagnosed as $\mathrm{CAD}$, and the number of significantly stenosed coronary vessels (VD), Gensini score and coronary artery calcification (CAC) score were quantified.
\end{abstract}

Results: There were no significant differences in age, high-density lipoprotein cholesterol, rates of hypertension, hemoglobin A1c, blood sugar or systolic blood pressure among the Low, Middle and High LDL-C groups. On the other hand, there were significant differences in rates of males, smoking, dyslipidemia and diabetes, diastolic blood pressure and triglyceride among the groups. The preva-

Manuscript submitted October 6, 2020, accepted November 4, 2020

Published online December 11, 2020

aDepartment of Cardiology, Fukuoka University Nishijin Hospital, Fukuoka, Japan

bepartment of Cardiology, Fukuoka University School of Medicine, Fukuoka, Japan

${ }^{\mathrm{c}}$ These authors contributed equally to this manuscript.

${ }^{\mathrm{d} C}$ Corresponding Author: Shin-ichiro Miura, Department of Cardiology, Fukuoka University School of Medicine, 7-45-1 Nanakuma, Jonan-ku, Fukuoka 814-0180, Japan. Email: miuras@cis.fukuoka-u.ac.jp

doi: https://doi.org/10.14740/cr1180 lence of CAD values in the Low, Middle and High LDL-C groups were similar, at $52 \%, 47 \%$, and $46 \%$, respectively. In addition, there were no significant differences in the number of VD, Gensini score or CAC score among the Low LDL-C, Middle LDL-C and High LDL-C groups.

Conclusions: We showed that the level of LDL-C was not associated with the presence or severity of CAD, which indicates that we need to screen by CCTA to prevent primary coronary events even if patients without anti-lipidemic therapies show low levels of LDL-C.

Keywords: Low-density lipoprotein cholesterol; Coronary artery disease; Coronary computed tomography angiography; Gensini score

\section{Introduction}

The incidence of atherosclerotic cardiovascular disease (ASCVD) is high when low-density lipoprotein cholesterol (LDLC) and/or triglyceride (TG) levels are high and/or when the high-density lipoprotein cholesterol (HDL-C) level is low [15]. According to the Japan Atherosclerosis Society Guidelines for the Prevention of Atherosclerotic Cardiovascular Diseases 2017 [6], the management target for LDL-C for primary prevention in patients with low, moderate and high coronary risk should be $<160 \mathrm{mg} / \mathrm{dL},<140 \mathrm{mg} / \mathrm{dL}$ and $<120 \mathrm{mg} / \mathrm{dL}$, respectively. Moreover, the target LDL-C for secondary prevention in patients with a history of coronary artery disease (CAD) is < $100 \mathrm{mg} / \mathrm{dL}$. For patients who have high-risk conditions, such as familial hypercholesterolemia, acute coronary syndrome or diabetes complicated by other high-risk conditions, the target LDL-C is $<70 \mathrm{mg} / \mathrm{dL}$.

We have been studying the Coronary Computed Tomography Angiography (FU-CCTA) Registry at Fukuoka University in Japan and have identified coronary risk factors for the primary prevention of CAD [7-16]. In this Registry, none of the patients were diagnosed with CAD until the time of CCTA because we used CCTA to screen for CAD. When patients had significant coronary stenosis as assessed by CCTA, they were 
initially diagnosed with CAD.

Therefore, using the FU-CCTA Registry, we hypothesized that the target LDL-C level for primary prevention of coronary events in patients should be $<70 \mathrm{mg} / \mathrm{dL}$ or $<100 \mathrm{mg} / \mathrm{dL}$.

\section{Materials and Methods}

\section{Study subjects}

One thousand sixteen consecutive subjects who were clinically suspected of having CAD or who had at least one cardiac risk factor (aging, gender male, hypertension (HTN), dyslipidemia (DL), diabetes mellitus (DM), smoking, obesity, family history of $\mathrm{CAD}$ and/or chronic kidney disease (CKD)) underwent CCTA. Suspected CAD is defined as having some chest symptoms and/or electrocardiogram (ECG) abnormalities. All subjects underwent CCTA between 2012 and 2019. Patients with creatinine $>2.0 \mathrm{mg} / \mathrm{dL}$ or contrast-induced allergy did not undergo multidetector computed tomography (MDCT). This study was conducted in compliance with the ethical standards of the responsible institution on human subjects as well as with the Helsinki Declaration. The protocol in this study was approved by the ethics committee of Fukuoka University Hospital (\#09-10-02), and all subjects gave their written informed consent to participate.

\section{Evaluation of coronary stenosis using CCTA}

We evaluated coronary stenosis using CCTA as previously described [7-9]. Patients were assessed by 64-MDCT on an Aquilion 64 (TOSHIBA, Tokyo, Japan) or by 320-MDCT on an Aquilion ONE ViSION (TOSHIBA, Tokyo, Japan). Overall, 15 coronary artery segments were assessed in all patients. Narrowing of the normal contrast-enhanced lumen to $\geq 50 \%$ that could be identified in multiplanar reconstructions or cross-sectional images was defined as significant stenosis [17]. The narrowing of the normal contrast-enhanced lumen to $\geq 70 \%$ was also defined as significant stenosis. When patients had significant coronary stenosis, they were initially diagnosed with CAD. In addition, in all patients, the severity of CAD was assessed in terms of the number of significantly stenosed coronary vessels (VD) and the Gensini score [18]. The coronary artery calcification (CAC) score was defined on CT images as the presence of more than two contiguous pixels with greater than 130 Hounsfield units. The CAC score in each lesion was then computed by the Agatston method [19].

\section{Evaluation of risk factors for CAD}

Body mass index (BMI), systolic blood pressure (SBP), diastolic BP (DBP), serum levels of TG, HDL-C, LDL-C, fasting blood glucose (FBG), hemoglobin A1c (HbA1c), smoking status, and medication use were collected as risk factors in all patients. BMI was calculated as weight $(\mathrm{kg}) /$ height $(\mathrm{m})^{2}$. The characteristics of patients were obtained from medical records

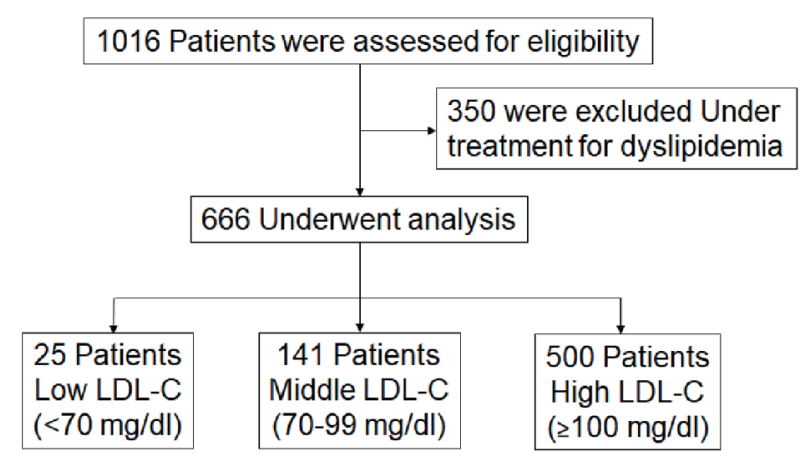

Figure 1. Flow chart of the enrollment process. LDL-C: low-density lipoprotein cholesterol.

with regard to history of HTN, DL and DM.

\section{Medications}

Information on medication use was obtained from medical records and included biguanide, dipeptidyl peptidase-4 inhibitor (DPP-4I), insulin, angiotensin II receptor blocker/angiotensinconverting enzyme inhibitor (ARB/ACEI), calcium channel blocker (CCB), $\beta$-blocker and diuretics.

\section{Statistical analysis}

A statistical analysis was performed using Excel 2016 (SSRI, Tokyo, Japan) and the Stat View statistical software package (Stat View 5; SAS Institute Inc., Cary, NC, USA). Continuous variables are shown as the mean \pm standard deviation. Categorical and continuous variables were analyzed for trends among the groups by the Cochran-Armitage trend test and Jonckheere-Terpstra trend test, respectively. A value of $\mathrm{P}<$ 0.05 was considered significant.

\section{Results}

\section{Patient characteristics}

One thousand sixteen patients were assessed for eligibility (Fig. 1). Three hundred fifty patients were excluded because they were receiving anti-lipidemic therapies. Six hundred sixty-six patients were divided into three groups: LDL-C $<70$ $\mathrm{mg} / \mathrm{dL}$ ( $\mathrm{n}=25$, Low LDL-C group), $100 \mathrm{mg} / \mathrm{dL}>$ LDL-C $\geq 70$ $\mathrm{mg} / \mathrm{dL}(\mathrm{n}=141$, Middle LDL-C group) and LDL-C $\geq 100 \mathrm{mg} /$ $\mathrm{dL}(\mathrm{n}=500$, High LDL-C group) (Table 1). The mean levels of LDL-C in the Low LDL-C, Middle LDL-C and High LDL-C groups were $57 \mathrm{mg} / \mathrm{dL}, 87 \mathrm{mg} / \mathrm{dL}$ and $133 \mathrm{mg} / \mathrm{dL}$, respectively ( $\mathrm{P}$ for trend $<0.001$ ). In addition, the mean levels of TG in the Low LDL-C, Middle LDL-C and High LDL-C groups were $125 \mathrm{mg} / \mathrm{dL}, 132 \mathrm{mg} / \mathrm{dL}$ and $136 \mathrm{mg} / \mathrm{dL}$, respectively ( $\mathrm{P}$ for trend $<0.001)$. There were no significant differences in age, HDL-C, rate of HTN, HbAlc, FBS, SBP or medications (the 
Table 1. Patients Characteristics in All Patients, Low LDL-C, Middle LDL-C and High LDL-C groups

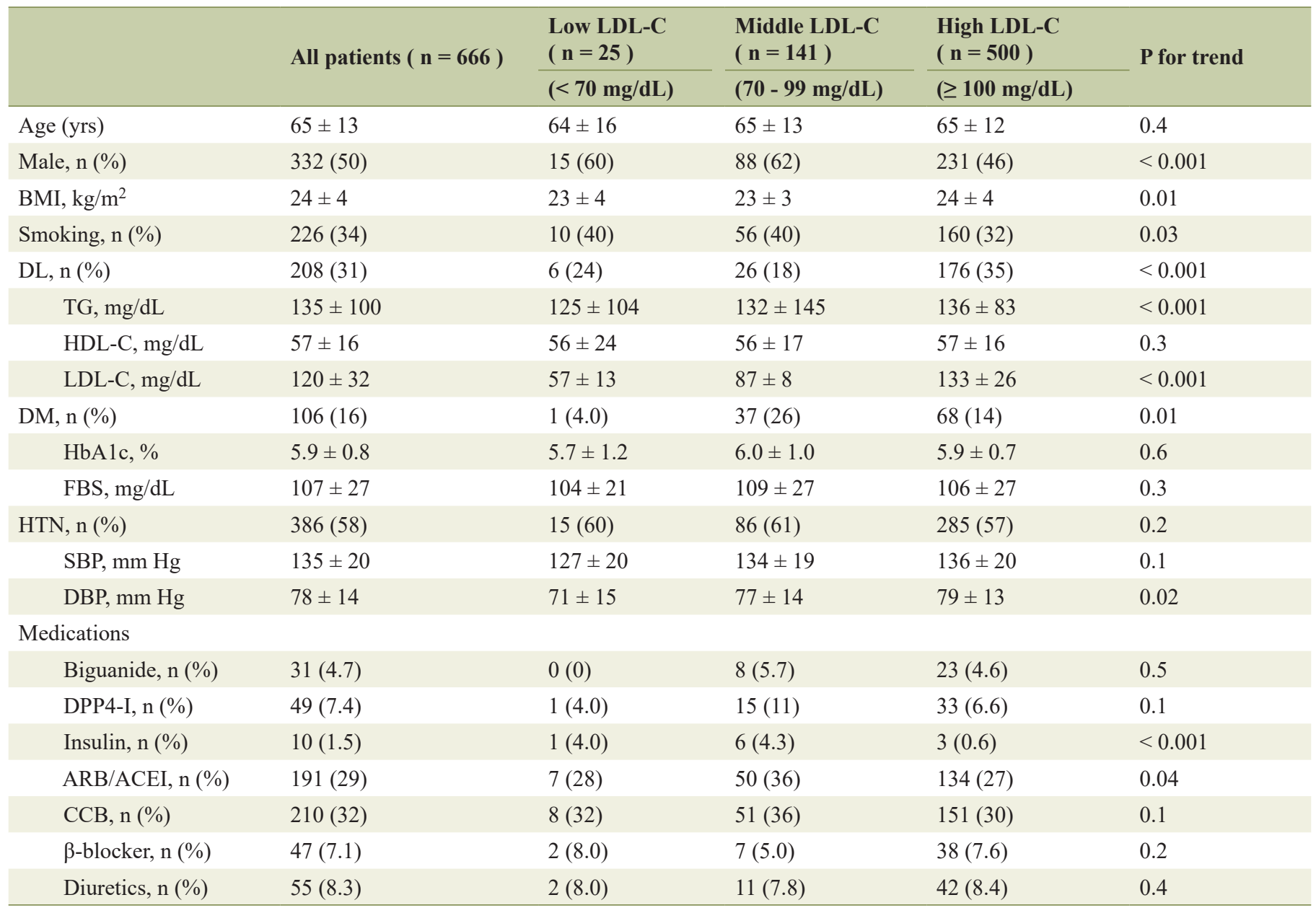

Continuous variables are expressed as mean \pm SD. BMI: body mass index; DL: dyslipidemia; TG: triglyceride; HDL-C: high-density lipoprotein cholesterol; LDL-C: low-density lipoprotein cholesterol; DM: diabetes mellitus; HbA1c: hemoglobin A1c; FBS: fasting blood sugar; HTN: hypertension; SBP: systolic blood pressure; DBP: diastolic blood pressure; DPP4-I: dipeptidyl peptidase 4 inhibitor. ARB/ACEI: angiotensin II receptor blocker/ angiotensin-converting enzyme inhibitor; CCB: calcium channel blocker.

rates of biguanide, DPP4-I, CCB, $\beta$-blocker and diuretics). On the other hand, there were significant differences in the rates of males, smoking, DL, DM, insulin and ARB/ACEI, and BMI and DBP.

\section{The prevalence of CAD, number of $\mathrm{VD}$, Gensini score and CAC score}

When $\geq 50 \%$ of coronary stenosis was defined as significant stenosis, the prevalence of CAD values in the Low LDL-C, Middle LDL-C and High LDL-C groups were similar, at 52\%, $47 \%$, and $46 \%$, respectively ( $\mathrm{P}$ for trend 0.3 ) (Fig. $2 \mathrm{a}$ ). The number of VD in the Low LDL-C, Middle LDL-C and High LDL-C groups was $0.8,0.9$ and 0.8 , respectively (P for trend 1.0) (Fig. 2b). The Gensini score and CAC score were also similar between the groups (Fig. 2c, d). In addition, when $\geq$ $70 \%$ of coronary stenosis was defined as significant stenosis, there were also no differences in $\% \mathrm{CAD}$ and the number of
VD among Low, Middle and High LDL-C groups (Fig. 2e, f).

\section{Discussion}

In this study, we hypothesized that the target LDL-C level in patients for the primary prevention of CAD events should be $<$ $70 \mathrm{mg} / \mathrm{dL}$ or $<100 \mathrm{mg} / \mathrm{dL}$. We showed that LDL-C $<70 \mathrm{mg} /$ $\mathrm{dL}$ or $<100 \mathrm{mg} / \mathrm{dL}$ under no anti-lipidemic therapies was not associated with the presence or severity of CAD. These results indicated that we need to screen with CCTA to prevent primary events regardless of LDL-C levels.

The most important finding in this study was that LDL-C $<70 \mathrm{mg} / \mathrm{dL}$ or $<100 \mathrm{mg} / \mathrm{dL}$ was not associated with lower the prevalence of CAD or a reduced severity of CAD, since there were no significant differences in the prevalence of $\mathrm{CAD}$, number of $\mathrm{VD}$, Gensini score or CAC score among the Low LDL-C, Middle LDL-C and High LDL-C groups. In a word, patients with lower LDL-C levels do not have a lower 
a. $\% \mathrm{CAD}$
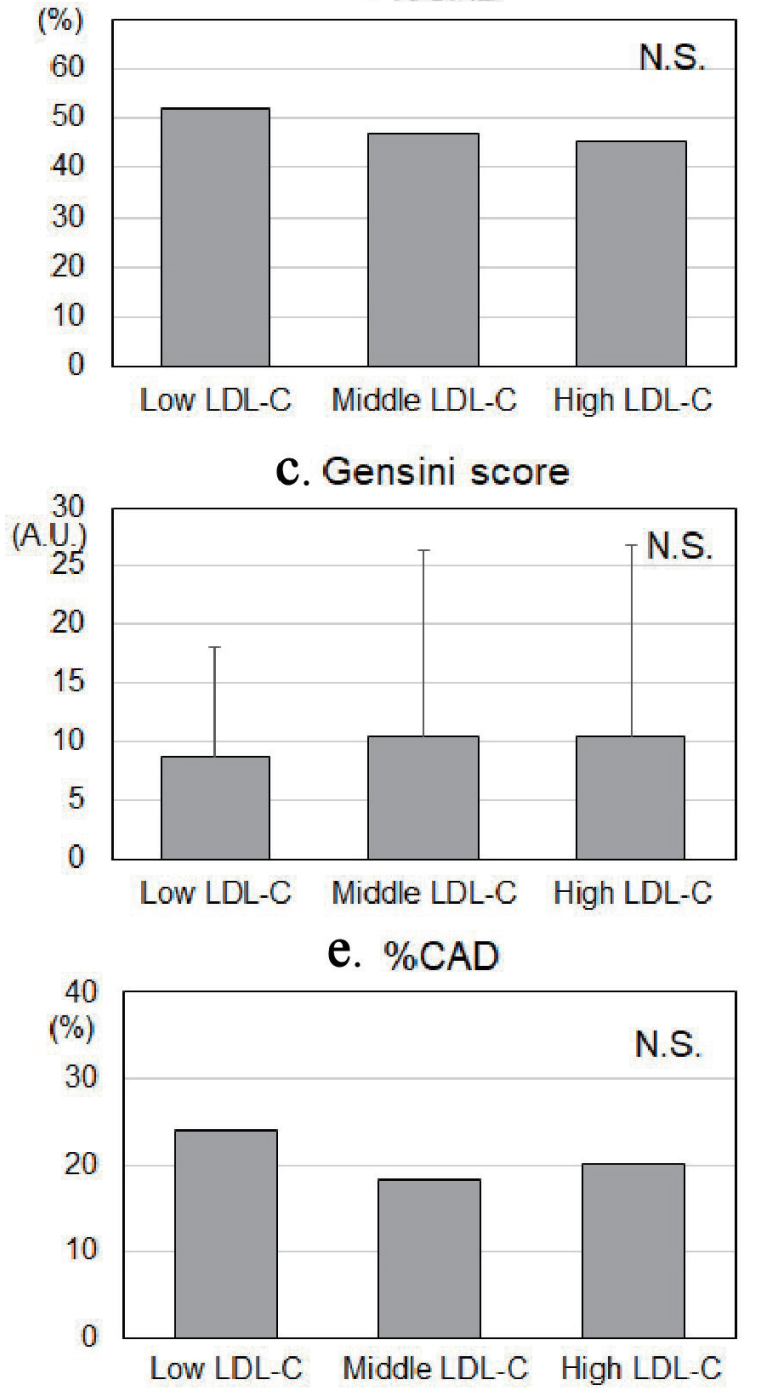

b. The number of VD
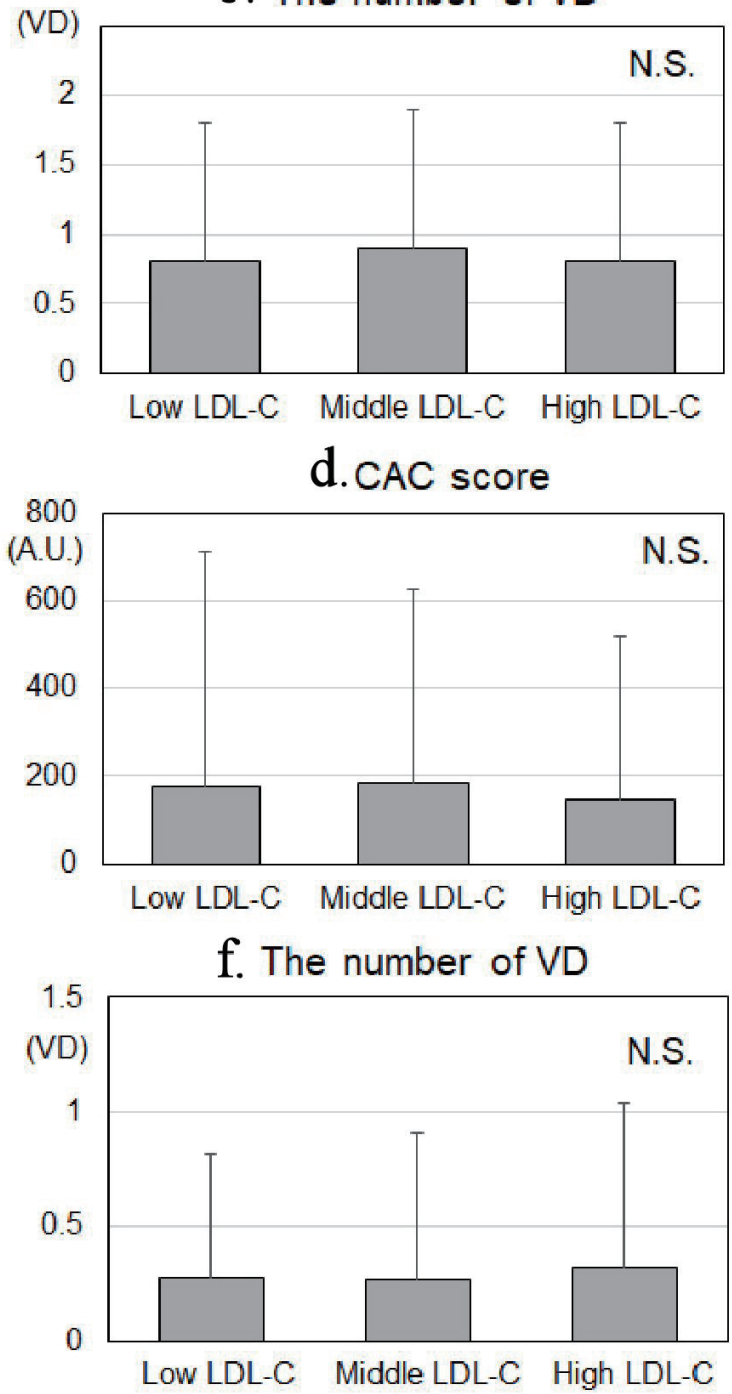

Figure 2. The prevalence of CAD (a, e), number of VD (b, f), Gensini score (c) and CAC score (d) in the Low LDL-C, Middle LDL$C$ and High LDL-C groups. The $\geq 50 \%$ and $\geq 70 \%$ of coronary stenosis were defined as significant stenosis in (a, b) and (e, f), respectively. CAD: coronary artery disease; number of VD: number of significantly stenosed coronary vessels; CACS: coronary artery calcium score; LDL-C: low-density lipoprotein cholesterol; N.S.: not significant; A.U.: arbitrary unit.

risk of CAD compared to patients with higher LDL-C $(\geq 100$ $\mathrm{mg} / \mathrm{dL})$. The High LDL-C group had lower rates of males, smoking and HTN compared to the Low and Middle LDL$\mathrm{C}$ groups. Male, smoking and HTN are critical coronary risk factors [20]. Although there were no significant differences in $\mathrm{HbA} 1 \mathrm{c}$ between the groups, the rate of insulin use in the High LDL-C group was lower than those in the Low and Middle LDL-C groups. The data indicated that the severity of DM in the High LDL-C group may be lower than those in the Low and Middle LDL-C groups. Although LDL-C levels in the High LDL-C group were relatively high $(\geq 100 \mathrm{mg} / \mathrm{dL})$, the rates of other risk factors were relatively low. Therefore, there were no significant differences in the presence or severity of CAD among the groups. In this study, patients who were clinically suspected of having CAD or who had at least one car- diac risk factor underwent CCTA. Thus, even though patients may show low LDL-C levels under no treatment of DL, it may be advisable to aggressively perform CCTA when patients are clinically suspected of having CAD or coronary risk factors other than DL.

This study has several important limitations. First, this study was cross-sectional. Second, CCTA is not a gold standard for the evaluation of CAD, although recent studies have shown that its sensitivity and specificity were both approximately $95 \%$ of those for invasive coronary angiography for the identification of significant coronary stenosis [21]. A largescale prospective study will be needed to address these issues.

In conclusion, the LDL-C level was not associated with atherosclerotic CAD, which indicates that we need to perform CCTA for screening to prevent primary cardiovascular events 
even if patients without anti-lipidemic therapies show low levels of LDL-C.

\section{Acknowledgments}

We thank all of the members of the Department of Cardiology, Fukuoka University Hospital, and Department of Cardiology, Fukuoka University Nishijin Hospital, Fukuoka, Japan.

\section{Financial Disclosure}

None to declare.

\section{Conflict of Interest}

None to declare.

\section{Informed Consent}

Informed consents were obtained.

\section{Author Contributions}

Conceptualization: Y. Katsuda and S.M. Validation: H.I., K.T., Y. Kawahira, and Y.I. Formal analysis: Y.S. and T.K. Investigation: M.S., H.N., and Y. Kawahira. Data curation: H.I., K.T., and Y.S. Writing-original draft: H.I., T.K., Y.S., and M.S. Writing-review and editing: S.M. Supervision: S.M.

\section{Data Availability}

The authors declare that data supporting the findings of this study are available within the article.

\section{References}

1. Nakamura H, Arakawa K, Itakura H, Kitabatake A, Goto Y, Toyota T, Nakaya N, et al. Primary prevention of cardiovascular disease with pravastatin in Japan (MEGA Study): a prospective randomised controlled trial. Lancet. 2006;368(9542):1155-1163.

2. Ridker PM, Danielson E, Fonseca FA, Genest J, Gotto AM, Jr., Kastelein JJ, Koenig W, et al. Rosuvastatin to prevent vascular events in men and women with elevated C-reactive protein. N Engl J Med. 2008;359(21):21952207.

3. LaRosa JC, Grundy SM, Waters DD, Shear C, Barter P, Fruchart JC, Gotto AM, et al. Intensive lipid lowering with atorvastatin in patients with stable coronary disease. N Engl J Med. 2005;352(14):1425-1435.

4. Pedersen TR, Faergeman O, Kastelein JJ, Olsson AG,
Tikkanen MJ, Holme I, Larsen ML, et al. High-dose atorvastatin vs usual-dose simvastatin for secondary prevention after myocardial infarction: the IDEAL study: a randomized controlled trial. JAMA. 2005;294(19):24372445.

5. Cannon CP, Braunwald E, McCabe CH, Rader DJ, Rouleau JL, Belder R, Joyal SV, et al. Intensive versus moderate lipid lowering with statins after acute coronary syndromes. N Engl J Med. 2004;350(15):1495-1504.

6. Kinoshita M, Yokote $\mathrm{K}$, Arai $\mathrm{H}$, Iida M, Ishigaki $\mathrm{Y}$, Ishibashi S, Umemoto S, et al. Japan Atherosclerosis Society (JAS) Guidelines for Prevention of Atherosclerotic Cardiovascular Diseases 2017. J Atheroscler Thromb. 2018;25(9):846-984.

7. Mitsutake R, Miura S, Kawamura A, Saku K. Are metabolic factors associated with coronary artery stenosis on MDCT? Circ J. 2009;73(1):132-138.

8. Mitsutake R, Miura S, Zhang B, Saku K. HDL-associated factors provide additional prognostic information for coronary artery disease as determined by multi-detector row computed tomography. Int J Cardiol. 2010;143(1):72-78.

9. Mitsutake R, Miura S, Shiga Y, Uehara Y, Saku K. Association between hypertension and coronary artery disease as assessed by coronary computed tomography. J Clin Hypertens (Greenwich). 2011;13(3):198-204.

10. Shiga Y, Miura S, Mitsutake R, Kawamura A, Uehara Y, Saku K. Significance of serum high-density lipoprotein cholesterol levels for diagnosis of coronary stenosis as determined by MDCT in patients with suspected coronary artery disease. J Atheroscler Thromb. 2010;17(8):870878.

11. Nakamura A, Miura S, Sugihara M, Miyase Y, Norimatsu $\mathrm{K}$, Shiga $\mathrm{Y}$, Nishikawa $\mathrm{H}$, et al. Contrast between innovator drug- and generic drug-induced renal dysfunction on coronary angiography (CONTRAST study). Heart Vessels. 2014;29(5):603-610.

12. Norimatsu K, Miura S, Suematsu Y, Shiga Y, Miyase Y, Nakamura A, Yamada M, et al. Associations between glycated albumin or hemoglobin A1c and the presence of coronary artery disease. J Cardiol. 2015;65(6):487-493.

13. Yano M, Miura S, Shiga Y, Miyase Y, Suematsu Y, Norimatsu K, Nakamura A, et al. Association between smoking habits and severity of coronary stenosis as assessed by coronary computed tomography angiography. Heart Vessels. 2016;31(7):1061-1068.

14. Norimatsu K, Kuwano T, Miura SI, Shimizu T, Shiga Y, Suematsu Y, Miyase Y, et al. Significance of the percentage of cholesterol efflux capacity and total cholesterol efflux capacity in patients with or without coronary artery disease. Heart Vessels. 2017;32(1):30-38.

15. Ueda Y, Shiga Y, Idemoto Y, Tashiro K, Motozato K, Koyoshi R, Kuwano T, et al. Association between the presence or severity of coronary artery disease and pericardial fat, paracardial fat, epicardial fat, visceral fat, and subcutaneous fat as assessed by multi-detector row computed tomography. Int Heart J. 2018;59(4):695-704.

16. Nose D, Shiga Y, Ueda Y, Idemoto Y, Tashiro K, Suematsu Y, Kuwano T, et al. Association between plasma levels of PCSK9 and the presence of coronary artery disease in 
Japanese. Heart Vessels. 2019;34(1):19-28.

17. Leipsic J, Abbara S, Achenbach S, Cury R, Earls JP, Mancini GJ, Nieman K, et al. SCCT guidelines for the interpretation and reporting of coronary $\mathrm{CT}$ angiography: a report of the Society of Cardiovascular Computed Tomography Guidelines Committee. J Cardiovasc Comput Tomogr. 2014;8(5):342-358.

18. Gensini GG. A more meaningful scoring system for determining the severity of coronary heart disease. Am J Cardiol. 1983;51(3):606.

19. Agatston AS, Janowitz WR, Hildner FJ, Zusmer NR, Viamonte M, Jr., Detrano R. Quantification of coronary ar- tery calcium using ultrafast computed tomography. J Am Coll Cardiol. 1990;15(4):827-832.

20. Umemura S, Arima H, Arima S, Asayama K, Dohi Y, Hirooka Y, Horio T, et al. The Japanese Society of Hypertension Guidelines for the Management of Hypertension (JSH 2019). Hypertens Res. 2019;42(9):1235-1481.

21. Ropers D, Rixe J, Anders K, Kuttner A, Baum U, Bautz W, Daniel WG, et al. Usefulness of multidetector row spiral computed tomography with 64- x 0.6-mm collimation and 330-ms rotation for the noninvasive detection of significant coronary artery stenoses. Am J Cardiol. 2006;97(3):343-348. 